\title{
FEA Analysis on Torsion Vibration Mode for Microdrills
}

\author{
Yanhong Sun ${ }^{\mathrm{a}}$, Ying Chen ${ }^{\mathrm{b}^{*}}$ and Feilong Wang ${ }^{\mathrm{c}}$ \\ College of Mechanical Engineering, Jilin Engineering Normal University, Changchun 130052, \\ China

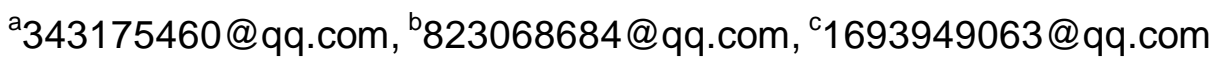 \\ ${ }^{*}$ The corresponding author
}

Keywords: Model; Microdrills; Torsion vibration; Modal analysis; FEA(finite element analysis)

\begin{abstract}
Microdrills' torsion vibration modes including the natural frequencies and modal shapes were studied by the theories on interspace kinematics, mechanism vibration and elastodynamics; Based on the theory analysis, a 3D model of micro-drill was designed by the $\operatorname{Pr} o /$ Engineer software, its torsion vibration modes were simulated by the MSC.FEA software, and some microdrills' dynamical characteristics on torsion vibration following its structure were illustrated, the change rule was talked about the natural frequencies with the overhang lengths of microdrills. The results indicate that the microdrills' modal shapes of the simulations and the theory models are completely consistent, the changing rules of the natural frequencies are same. The calculating results verified fully their correctness and practicalness on this paper's mathematics models. Through the analysis of the torsional vibration mode of microdrill, it can provide the reference for the effective measures to prevent the drill bit from breaking during the drilling process.
\end{abstract}

\section{Introduction}

With the development of science and technology are increasingly miniaturization, integration, high precision, micro processing more and more, The mechanical drilling with micro twist drill is widely used because of its low cost, high efficiency, good precision and simple operation[1]. However, because of the high speed rotation of the microdrill, it is often easy to cause the resonance, which affects the machining precision and the quality of the product. In order to facilitate the analysis and study on vibration analysis of the microdrill, it can be divided into 3 parts: torsional vibration, flexural vibration and longitudinal vibration[2].

In this paper, the theory of space kinematics[3], mechanical vibration and elastic dynamics are used to study the torsional vibration of microdrill. To understand and grasp the microdrill torsional vibration natural frequency and corresponding vibration mode modal parameters, not only conducive to the rational selection of machining parameters in the machining process[4], and to further suppress torsional vibration and improve the service life and provide a theoretical basis of microdrill.

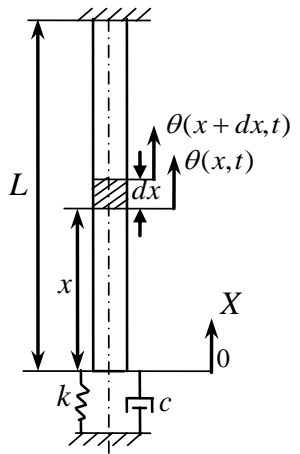

Figure 1. torsion vibration frame of microdrill

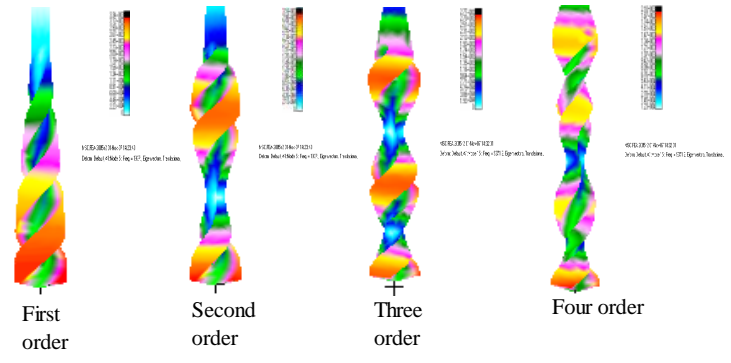

Figure 2. torsion vibration modal shapes 


\section{Torsion Vibration Mode of Microdrill}

Basic Assumptions. The microdrill bit is a homogeneous elastic straight rod[5], and the diameter of the spiral chip groove has little difference with the clamping part; It is assumed that the material is linear elastic and does not yield; The microdrill axis coincides with the axis of the machine tool, ignoring the bending deformation of the microdrill and the friction with the wall; The input speed of the bit is constant; The influence of temperature field is not considered in the drilling process[6]; The microdrill is affected by the torque to produce relative angular displacement.

Establishment of Mathematical Model. As shown in Fig.1, the length of microdrill is $L$, When the torsion is reversed, each cross section rotates an angle around the axis of the cross section to $\theta$, Cross section remains plane. The torsional angle $\theta$ is not only dependent on the space coordinates $x$, but also depends on the time of $t$, and the displacement of each point on the cross section is determined by the torsion angle of the section. The torsion angle $\theta$ can be used as the generalized coordinates of the cross section. At the cross section of $x$, the length of the length of the shaft of the moment of inertia of $J(x)$ and the moment of inertia of the cross section of the shaft is a function of the coordinates of the $x$, the shear modulus of elasticity is $G$, the length of the shaft is about $d x$, and the shear modulus is about $I_{p}(x)$.

Based on D Alembert principle[3] listed in homogeneous elastic straight rod partial differential equations of torsional vibration[7]

$$
J \frac{\partial^{2} \theta(x, t)}{\partial t^{2}}-G I_{p} \frac{\partial^{2} \theta(x, t)}{\partial x^{2}}=f(x, t)
$$

This equation is a partial differential equation for torsional vibration of microdrill.

If $f(x, t)=0$ is the partial differential equation of the free vibration of the microdrill[8].

$$
J \frac{\partial^{2} \theta(x, t)}{\partial t^{2}}-G I_{p} \frac{\partial^{2} \theta(x, t)}{\partial x^{2}}=0
$$

By using the method of separation of variables, Set $\theta(x, t)=\Theta(x) F(t)$, the micro displacement of the drill bit vibration is $\Theta(x)$, only depends on the variable of $x, F(t)$, said the drill bit vibration law, only depends on the time of $t$, into the equation (2);

$$
J \Theta(x) \frac{\partial^{2} F(t)}{\partial t^{2}}-G I_{p} F(t) \frac{\partial^{2} \Theta(x)}{\partial x^{2}}=0
$$

Get

$$
\begin{aligned}
& \frac{\Theta^{\prime \prime}(x)}{\Theta(x)}=\frac{J}{G I_{p}} \frac{F^{\prime \prime}(t)}{F(t)}=-\lambda^{2} \\
& \left\{\begin{array}{l}
F^{\prime \prime}(t)+\frac{\lambda^{2} G I_{p}}{J} F(t)=0 \\
\Theta^{\prime \prime}(x)+\lambda^{2} \Theta(x)=0 \\
\omega^{2}=\frac{G I_{p}}{J} \lambda^{2}
\end{array}\right.
\end{aligned}
$$

The general solution of the equation is

$$
\begin{aligned}
& \left\{\begin{array}{l}
F(t)=A \sin \omega t+B \cos \omega t \\
\Theta(x)=C \sin \lambda x+D \cos \lambda x
\end{array}[9]\right. \\
& \omega=\lambda \sqrt{\frac{G I_{p}}{J}} \text { 。 }
\end{aligned}
$$

The natural frequency of the bit can be obtained by the boundary condition

$$
\Theta(0)=0 ; \Theta^{\prime}(L)=0, J=\frac{1}{8} m D^{2}, I_{p}=\frac{\pi D^{4}}{32}, \omega_{r}=\frac{(2 r-1) \pi \sqrt{\frac{G I_{p}}{J}}}{2 L}=\frac{(2 r-1) \pi \sqrt{\frac{G}{\rho}}}{2 L} \quad(r=1,2, \ldots)
$$


The corresponding mode function;

$$
\Theta_{r}(x)=\sin \frac{(2 r-1) \pi}{2 L} x \quad(r=1,2, \ldots)
$$

\section{Finite Element Calculation of Torsional Vibration Mode of Microdrill}

Finite element analysis of the structure, there are three main steps: to create a finite element model (pre-treatment) to set the material properties and solve (solution) to view the results of calculation and animation (post-processing)[10].

Test Parameters. Microdrill geometry parameters: diameter $0.4 \mathrm{~mm}$, total length $20 \mathrm{~mm}$, spiral slot part length of $5 \mathrm{~mm}$, drill core diameter $=0.128 \mathrm{~mm}$; Main angle: Helix angle $\beta=19^{\circ}$, Tip angle $2 \phi=118^{\circ}$,Chisel edge angle $\psi=55^{\circ}$; Mechanical properties of microdrill materials: Strength limit $[\tau]=920 \mathrm{MPa}$,Elastic modulus $G=80 \mathrm{GPa}$,Material density $\rho=8300 \mathrm{~kg} / \mathrm{m}^{3}$; Cutting dosage: Feed quantity: $f=40 \mathrm{~mm} / \mathrm{min}$,Spindle speed $n=15000 \mathrm{r} / \mathrm{min}, 22000 \mathrm{r} / \mathrm{min}, 30000 \mathrm{r} / \mathrm{min}$; Workpiece material for stainless steel[1].

Torsional Vibration Mode of Microdrill. Pro/E software is used to design the 3D solid model of microdrill, and the finite element analysis of microdrill is made by MSC.FEA software. The boundary constraint of the top part of the microdrill head can be simulated as a fixed end, while the microdrill is only allowed to rotate around the axis. When the length of the drill bit is $L=7 \mathrm{~mm}$, the torsional vibration mode of the first four bits is extracted, and the natural frequencies are shown in Table 1, and the vibration modes of each mode are shown in Fig. 2.

Table 1 Natural frequencies of torsional vibration of microdrills [rad/s]

\begin{tabular}{ccc}
\hline Order number & Theoretical value of natural frequency & Simulation result \\
\hline 1 & 19156 & 22030 \\
2 & 62547 & 66092 \\
3 & 90248 & 110153 \\
4 & 134210 & 154214 \\
\hline
\end{tabular}

\section{Solution Results and Analysis}

Natural Frequency Analysis. In order to verify the correctness of the model, the equation (5) is calculated, and the first natural frequency of the torsional vibration is obtained. The results are compared with the results of the modal calculation, as shown in Table 1 and Fig.3. The change tendency of the torsional vibration frequency of the microdrill model and the actual model is similar to that of the actual model; Because of the influence of the spiral groove structure of the drill bit on its quality, rigidity, strength and so on[11], the natural frequency and the theoretical value are different.

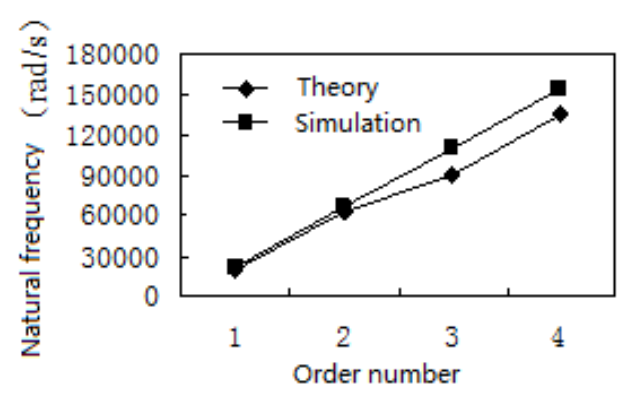

Figure 3. Comparison of natural frequency

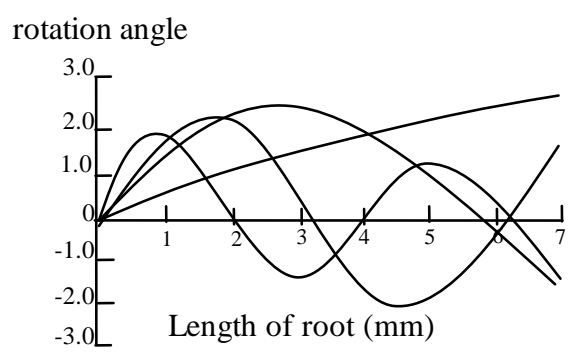

Figure 4. former four level modal shapes

Vibration Mode Analysis. The results of the various types of vibration data extracted from the graph drawn together. As shown in Fig. 4. The figure shows that the maximum torsion angle of microdrill the first-order torsional vibration occurs in the drill point, a large head and a small head vibration; The maximum angular displacement of two order torsional vibration is located away from the clamping root $3.5 \mathrm{~mm}$, negative in drill point, microdrill in the upper and the drill has large torsional; The maximum torsional angle of the three order torsional vibration of the microdrill is $2.0 \mathrm{~mm}$ from the root 
of the clamp, and the negative direction occurs at $4.5 \mathrm{~mm}$; Microdrill four order torsional vibration maximum torsion angle clamping root $1.0 \mathrm{~mm}$, negative in the drill point. Three order, the second order vibration modes are generated in the section of the cross torsion, that is, the opposite torsion angle of each section of the four. The torsional vibration mode of the bit is basically in accordance with the sine curve, which shows the correctness of the theoretical model.

\section{Conclusion}

Using the theory of space kinematics, mechanical vibration and Based on the principle of dynamic mechanics, the torsional vibration mode of microdrill is established. The model fully reflects the influence of the structure parameters and processing parameters on the natural frequency and vibration mode of the bit.

The design of three-dimensional geometric solid model of microdrill conforms to the actual structural characteristics which ensure the accuracy of the simulation results.

Through numerical simulation, to extract micro rank natural frequency of drill torsional vibration modes and the corresponding mode, and compared with the theoretical calculation results, illustrate the structural characteristics of the microdrill itself has a certain influence on the torsional vibration mode.

\section{References}

[1] Y.H. Sun: Research on Breakage Mechanism of Micro-drills and Drilling Forces Online Monitoring(Ph.D. Jilin University, China 2009),p.18-34(In Chinese)

[2] Magara T M,Y.C. Pei and Q.C. Tan: Study on the characteristics of eccentric drilling drill into microdrill [J].Journal of Jilin University(Engineering and Technology Edition),2005,No.8,p.61

[3] Y.M. Zhang; Mechanical Vibration [M]. Jilin Science \& Technology Publishing House,2000

[4] J.H. Fan, D.G. Zhang: B-spline interpolation method for dynamics of a rotating cantilever beam [J]. Chinese Journal of Mechanical Engineering, Vol. 23(2012),p.59-64(In Chinese)

[5] Y.N. Cheng, Y.N. Gong and Y. Han; Vibration modal analysis of cutter and main shaft system in heavy milling process of water chamber head [J]. Vibration and Shock, Vol.34(2015), No.18,p.39-44(In Chinese)

[6] J.J. Wang, J.G. Qian: Machine Dynamics [M]. China University of Mining \& Technology Press, 1993(In Chinese)

[7] K. An, Y. Bi and J.G. Ma: Vibration modal analysis of single link manipulator with end-effector payload [J]. Optoelectronic Engineering, Vol.43(2016),No.7,p.22-27(In Chinese)

[8] H.Z. L: Structural dynamic model for simulation of cutting vibration of machine tools [J]. Journal of Tongji University (Natural Science). 1995,No.5,p.123-126(In Chinese)

[9] F.G. Yan, G.H. Ho and X.L. Liu: Technology of highly efficient tool under the condition of extremely heavy load [J]. Journal of Harbin University of Science and Technology. 2011, No.6, p.42-46(In Chinese)

[10] C.Y. Jia: Reasons and solutions of NC tool chatte [J].Mechanical Engineering \& Automation. 2008,No.4,p.87-90(In Chinese)

[11]X.R. Han: Research on the Construction Dynamics Model of Cutting Vibration of Machine tool[J].Machine Tool \& Hydraulics.2003, No.4,p.57-61(In Chinese) 